\title{
Other Blood Form Product
}

National Cancer Institute

\section{Source}

National Cancer Institute. Other Blood Form Product. NCI Thesaurus. Code C133277.

Another blood form product type other than the ones that are explicitly identified in this value set. 